UCRHEP-T261

August 1999

\title{
Hierarchical Four-Neutrino Oscillations With a Decay Option
}

\author{
Ernest $\mathrm{Ma}^{1}$, G. Rajasekaran${ }^{2}$, Ion Stancu ${ }^{1}$ \\ ${ }^{1}$ Physics Department, Univeristy of California, Riverside, CA 92521, USA \\ ${ }^{2}$ Institute of Mathematical Sciences, Madras 600113, India
}

\begin{abstract}
We present a new and novel synthesis of all existing neutrino data regarding the disappearance and appearance of $\nu_{e}$ and $\nu_{\mu}$. We assume four neutrinos: $\nu_{e}, \nu_{\mu}, \nu_{\tau}$, as well as a heavier singlet neutrino $\nu_{s}$ of a few $\mathrm{eV}$. The latter may decay into a massless Goldstone boson (the singlet Majoron) and a linear combination of the doublet antineutrinos. We comment on how this scenario may be verified or falsified in future experiments.
\end{abstract}


Accepting the totality of present experimental evidence for neutrino oscillations [1, 2, 3], it is not unreasonable to entertain the idea that there are four light neutrinos. Since the invisible decay of the $Z$ boson tells us that there are only three light doublet neutrinos, i.e. $\nu_{e}, \nu_{\mu}, \nu_{\tau}$, the fourth light neutrino $\nu_{s}$ should be a singlet. Usually, $\nu_{s}$ is assumed to mix with the other neutrinos in a $4 \times 4$ mass matrix for a phenomenological understanding [4] of all the data. However, given that $\nu_{s}$ is different from $\nu_{e, \mu, \tau}$, it may have some additional unusual property, such as decay. In fact, as shown below, this is a natural consequence of the spontaneous breakdown of lepton number in the simplest model[5], and it has some very interesting and verifiable predictions in future neutrino experiments.

If only atmospheric[1] and solar[2] neutrino data are considered, then hierarchical threeneutrino oscillations with

$$
\begin{aligned}
& \nu_{1}=\nu_{e} \cos \theta-\frac{1}{\sqrt{2}}\left(\nu_{\mu}+\nu_{\tau}\right) \sin \theta, \\
& \nu_{2}=\nu_{e} \sin \theta+\frac{1}{\sqrt{2}}\left(\nu_{\mu}+\nu_{\tau}\right) \cos \theta, \\
& \nu_{3}=\frac{1}{\sqrt{2}}\left(-\nu_{\mu}+\nu_{\tau}\right),
\end{aligned}
$$

where $m_{1}<<m_{2}<<m_{3}$, would fit the data very well. Here $m_{3}^{2} \sim 10^{-3} \mathrm{eV}^{2},\left(\sin ^{2} 2 \theta\right)_{a t m}=1$, and $m_{2}^{2} \sim 10^{-5} \mathrm{eV}^{2}$ for the matter-enhanced oscillation solution 6 to the solar neutrino deficit with $\left(\sin ^{2} 2 \theta\right)_{\text {sol }} \sim 10^{-3}$ or near 1 , or $m_{2}^{2} \sim 10^{-10} \mathrm{eV}^{2}$ for the vacuum oscillation solution with $\left(\sin ^{2} 2 \theta\right)_{\text {sol }} \sim 1$.

We now add a fourth neutrino $\nu_{s}$ and assume that it mixes a little with $\nu_{e}$ and $\nu_{\mu}$ to explain the LSND data[3]. Since the relevant $\Delta m^{2}$ is now about $1 \mathrm{eV}^{2}$, it is natural to take $m_{4}^{2} \sim 1 \mathrm{eV}^{2}$, but this hierarchical solution is disfavored [7], because the observed $\bar{\nu}_{\mu} \rightarrow \bar{\nu}_{e}$ probability [3] is contradicted by the $\nu_{\mu} \rightarrow \nu_{\mu}$ data of CDHSW [8] together with the $\bar{\nu}_{e} \rightarrow \bar{\nu}_{e}$ data of Bugey [9]. However, there are two ways that this conclusion may be evaded. (1) Let $m_{4}^{2} \sim 25 \mathrm{eV}^{2}$, then the constraint due to the CDHSW experiment is not a factor, but now 
there are three other accelerator $\nu_{\mu} \rightarrow \nu_{e}$ experiments: BNL-E734[10], BNL-E776 [11], and CCFR 12], which have bounds close to but allowed by the LSND $99 \%$ likelihood contour. This is a marginal hierarchical four-neutrino oscillation solution to all the data. (2) If $\nu_{4}$ decays, then the parameter space for an acceptable solution should open up. For example, in the CDHSW experiment, two detectors at different distances compare their respective $\nu_{\mu}$ fluxes and the ratio is taken. If the $\nu_{4}$ component of $\nu_{\mu}$ decays away already before reaching the first detector, the ratio remains at unity. In contrast to the case of only oscillations, this experiment is then unable to restrict $m_{4}^{2}$. Not only that, since the argument[]] against the hierarchical four-neutrino spectrum depends crucially on the CDHSW experiment, it is clear that it cannot be valid in general.

The idea of neutrino decay is of course not new. It is naturally related to the spontaneous breakdown of lepton number [5, 13]. The associated massless Nambu-Goldstone boson[14] is called the Majoron and the typical decay $\nu_{2} \rightarrow \bar{\nu}_{1}+$ Majoron occurs if kinematically allowed. The triplet Majoron[13] is ruled out experimentally because the decay $Z \rightarrow$ Majoron + partner (imaginary and real parts respectively of the lepton-number carrying scalar field) would have counted as the equivalent of two extra neutrino flavors. The singlet Majoron [5] is unconstrained because it has no gauge interactions. We assign lepton number $L=-1$ to $\nu_{s}$ and assume the existence of a scalar particle $\chi^{0}$ with $L=2$. [By convention, $\nu_{s}$ is left-handed. If we use a right-handed singlet neutrino $\nu_{R}$ instead, then it would be assigned $L=+1$.] Hence the relevant terms of the interaction Lagrangian are given by

$$
\mathcal{L}_{i n t}=g_{s} \nu_{s} \nu_{s} \chi^{0}+\sum_{\alpha=e, \mu, \tau} h_{\alpha} \nu_{s}\left(\nu_{\alpha} \phi^{0}-l_{\alpha} \phi^{+}\right)+h . c .
$$

As $\left\langle\chi^{0}\right\rangle$ and $\left\langle\phi^{0}\right\rangle$ become nonzero, $\nu_{s}$ becomes massive and also mixes with $\nu_{e, \mu, \tau}$ to form the mass eigenstates $\nu_{1,2,3,4}$. At the same time, $\sqrt{2} \operatorname{Im} \chi^{0}$ becomes the massless Majoron $M$ and the decay

$$
\nu_{4} \rightarrow \bar{\nu}_{1,2,3}+M
$$


is now possible. Neutrino decay involving only $\nu_{e, \mu, \tau}$ was recently proposed 15 to explain the atmospheric data[1], but that becomes a poor fit after the inclusion of the upward going muons [16]. More recently, it was shown[17] that combining oscillation and decay (at the expense of also adding $\nu_{s}$ ) gives again a good fit. In contrast, the effects we envisage here of $\nu_{4}$ decay in atmospheric and solar neutrino data are both small and do not change the usual oscillation interpretation appreciably, as shown below.

Let $\nu_{e, \mu, \tau, s}$ be related to the mass eigenstates $m_{1,2,3,4}$ through the unitary matrix $U_{\alpha i}$, which will be assumed real in the following for simplicity. Let $m_{4}>>m_{3}>>m_{2}>>m_{1}$ with $\nu_{4}$ having the decay lifetime $\tau_{4}$. Then for solar and atmospheric neutrino oscillations with $m_{4}^{2} L / 4 E>>1$, the probability of $\nu_{\alpha} \rightarrow \nu_{\beta}$ is given by

$$
P_{\alpha \beta}=\delta_{\alpha \beta}\left(1-2 U_{\alpha 4}^{2}\right)+U_{\alpha 4}^{2} U_{\beta 4}^{2}\left(1+x^{2}\right)-4 \sum_{i<j<4} U_{\alpha i} U_{\alpha j} U_{\beta i} U_{\beta j} \sin ^{2} \frac{\Delta m_{i j}^{2} L}{4 E},
$$

where

$$
x=e^{-m_{4} L / 2 E \tau_{4}} .
$$

In the case of laboratory experiments where $\Delta m_{i j}^{2} L / 4 E<<1$ for $i<j<4$ but $m_{4}^{2} L / 4 E$ is not necessarily large or small, the corresponding formula is

$$
P_{\alpha \beta}=\delta_{\alpha \beta}\left[1-2 U_{\alpha 4}^{2}\left(1-x \cos \frac{m_{4}^{2} L}{2 E}\right)\right]+U_{\alpha 4}^{2} U_{\beta 4}^{2}\left[1-2 x \cos \frac{m_{4}^{2} L}{2 E}+x^{2}\right] .
$$

Note that the above expression simplifies to a function of $U_{\alpha 4}, U_{\beta 4}$, and $x$ if $m_{4}$ is large, and to a function of $U_{\alpha 4}$ and $U_{\beta 4}$ alone if $x=0$ whatever the value of $m_{4}$. In those circumstances, the corresponding laboratory experiment has no sensitivity to oscillations, but does measure one fixed number. Specifically, if $m_{4}$ is large, then

$$
P_{\mu e}=U_{e 4}^{2} U_{\mu 4}^{2}\left(1+x^{2}\right), \quad P_{e e}=\left(1-U_{e 4}^{2}\right)^{2}+x^{2} U_{e 4}^{4}, \quad P_{\mu \mu}=\left(1-U_{\mu 4}^{2}\right)^{2}+x^{2} U_{\mu 4}^{4} .
$$

If $x=0$, then regardless of $m_{4}$, Eq. (8) reduces to Eq. (9) but with $x$ set equal to zero. The 
LSND experiment obtains[3]

$$
P_{\mu e}=3.1+\frac{+1.1}{-1.0} \pm 0.5 \times 10^{-3}
$$

whereas BNL-E734 has 10 $P_{\mu e}<1.7 \times 10^{-3}$ and BNL-E776 has 11 Pe $P_{\mu e}<1.5 \times 10^{-3}$. Using the LSND $90 \%$ confidence-level limit of $P_{\mu e}>1.3 \times 10^{-3}$, we find therefore reasonable consistency among these experiments. [The most recent result of the ongoing KARMEN II experiment [18] is $P_{\mu e}<2.1 \times 10^{-3}$, which will eventually have the sensitivity to test Eq. (10).] The recent CCFR experiment[12] measures $P_{\mu e}<0.9 \times 10^{-3}$, but its average $L / E$ is one to two orders of magnitude smaller than those of the other experiments, hence its $x$-value may be taken to be close to one and the usual oscillation interpretation of the data holds. This constraint implies that $m_{4}^{2}<30 \mathrm{eV}^{2}$.

At $m_{4} \sim 5 \mathrm{eV}$, we are below the CCFR exclusion and in a marginal region of the parameter space for pure neutrino oscillations consistent with the LSND evidence and the exclusion from BNL-E734 and BNL-E776. Between $m_{4} \sim 5 \mathrm{eV}$ and $m_{4} \sim 3 \mathrm{eV}$, the BNLE734 data exclude a solution if $x=1$ and because that experiment has an average $L / E$ an order of magnitude smaller than that of BNL-E776, LSND, or CDHSW, the decay factor goes against having a consistent solution here even if $x<1$. Below $m_{4} \sim 3 \mathrm{eV}$, the oscillation + decay interpretation of the latter 3 experiments becomes important, as shown below.

Ideally, one should reanalyze the results of all the laboratory experiments using Eq. (8) and verify whether the positive LSND signal can coexist with the exclusion limits from the other laboratory experiments by extending the usual parameter space of $m_{4}, U_{e 4}$, and $U_{\mu 4}$ to include $\tau_{4}$ as well. This can be done only by using the full data set of each of the experiments and is best performed by the experimenters themselves. In the absence of such a calculation, we point out here the crucial fact that the CDHSW experiment 8 would see no difference in its two detectors at distances of $130 \mathrm{~m}$ and $885 \mathrm{~m}$, if the effective values of the quantity $\exp \left(-m_{4} L / 2 E \tau_{4}\right) \cos \left(m_{4}^{2} L / 2 E\right)$ is the same. In Table I, we show $\Gamma_{4} / m_{4}\left(=1 / \tau_{4} m_{4}\right)$ as a 
function of $m_{4}^{2}$ near $6 \mathrm{eV}^{2}$ for which this happens, using as our very crude approximation the fixed values of $L_{1} / E=0.065 \mathrm{~m} / \mathrm{MeV}$ and $L_{2} / E=0.442 \mathrm{~m} / \mathrm{MeV}$. This illustrates the possibility that the decrease from $x_{1}$ to $x_{2}$ due to decay may be compensated by the increase in the value of the cosine from $L_{1}$ to $L_{2}$ due to oscillations. Note also that there is a range of $m_{4}^{2}$ for which a null solution exists with varying $\Gamma_{4} / m_{4}$, whereas if the latter is zero, then $m_{4}^{2}$ has only discrete solutions (at 4.8 and $6.6 \mathrm{eV}^{2}$ for example). In the realistic case of integrating over the experimental energy spectrum, both solutions will be smeared out, but the possibility of decay should result in a larger range of acceptable values of $m_{4}^{2}$. For consistency, we also show in Table I the values of $f \equiv P_{\mu e} / U_{e 4}^{2} U_{\mu 4}^{2}=1-2 x \cos \left(m_{4}^{2} L / 2 E\right)+x^{2}$ for the LSND and BNL-E776 experiments, using the fixed values of $L / E=0.75$ and 0.5 $\mathrm{m} / \mathrm{MeV}$ respectively. This shows that the value of $P_{\mu e}$ as seen by the LSND experiment can be larger than that of BNL-E776 for $4.8<m_{4}^{2}<5.8 \mathrm{eV}^{2}$.

To discuss solar and atmospheric neutrino oscillations, let us focus on the following specific model. Let $\cos \theta=\sqrt{2 / 3}$ and $\sin \theta=\sqrt{1 / 3}$ in Eqs. (1) and (2), and let $\nu_{s}$ mix with $\nu_{2}$ only, then $U_{\alpha i}$ is given by

$$
U=\left[\begin{array}{cccc}
\sqrt{2 / 3} & c \sqrt{1 / 3} & 0 & s \sqrt{1 / 3} \\
-\sqrt{1 / 6} & c \sqrt{1 / 3} & -\sqrt{1 / 2} & s \sqrt{1 / 3} \\
-\sqrt{1 / 6} & c \sqrt{1 / 3} & \sqrt{1 / 2} & s \sqrt{1 / 3} \\
0 & -s & 0 & c
\end{array}\right]
$$

where $c$ and $s$ are respectively the cosine and sine of the $\nu_{s}-\nu_{2}$ mixing angle. For solar neutrino oscillations, we have

$$
P_{e e}=\left(1-\frac{s^{2}}{3}\right)^{2}-\frac{4}{9}\left(1-s^{2}\right)\left(1-\cos \frac{\Delta m_{12}^{2} L}{2 E}\right)+\frac{x^{2} s^{4}}{9} .
$$

In the limit $s=0$, this reduces to the usual two-neutrino formula with $\sin ^{2} 2 \theta=8 / 9$ which is a good fit to the data[2], either as the large-angle matter-enhanced solution or the vacuum oscillation solution. With a small $s^{2} / 3$ of order a few percent [between $0.026(x=1)$ and 
$0.037(x=0)$ for $\left.P_{\mu e}(\mathrm{LSND})=1.35 \times 10^{-3}\right]$, this is definitely still allowed. Note that this result is not sensitive at all to the last term because $s^{4} / 9$ is of order $10^{-3}$.

For atmospheric neutrino oscillations, we have

$$
\begin{gathered}
P_{e e}=\left(1-\frac{s^{2}}{3}\right)^{2}+\frac{x^{2} s^{4}}{9}, \quad P_{e \mu}=P_{\mu e}=\left(1+x^{2}\right) \frac{s^{4}}{9} \\
P_{\mu \mu}=\left(1-\frac{s^{2}}{3}\right)^{2}-\frac{1}{2}\left(1-\frac{2 s^{2}}{3}\right)\left(1-\cos \frac{\Delta m_{23}^{2} L}{2 E}\right)+\frac{x^{2} s^{4}}{9} .
\end{gathered}
$$

Here the limit $s=0$ corresponds to the canonical $\nu_{\mu} \rightarrow \nu_{\tau}$ solution with $\sin ^{2} 2 \theta=1$. As it is, the prediction of $\nu_{e} \rightarrow \nu_{e}$ is still a fixed number, but smaller than unity (0.93 for $\left.s^{2} / 3=0.037\right)$. Given that there is an uncertainty of about $20 \%$ in the absolute flux normalization, we should consider instead the ratio

$$
\frac{2 P_{\mu \mu}+P_{e \mu}}{P_{e e}+2 P_{\mu e}} \simeq 2\left[1-\frac{s^{4}}{6}-\frac{1}{2}\left(1-\frac{2 s^{4}}{9}\right)\left(1-\cos \frac{\Delta m_{23}^{2} L}{2 E}\right)\right],
$$

where we have made an expansion in powers of $s^{2}$ and assumed that the ratio of $\nu_{\mu}$ to $\nu_{e}$ produced in the atmosphere is two. It is clear that this is numerically indistinguishable from the case $s=0$.

In this model, the decay $\nu_{4} \rightarrow \bar{\nu}_{2}+M$ has some very interesting experimental consequences. For example, $\nu_{e}$ from the sun decays through its $\nu_{4}$ component into $\bar{\nu}_{2}=$ $(c / \sqrt{3})\left(\bar{\nu}_{e}+\bar{\nu}_{\mu}+\bar{\nu}_{\tau}\right)-s \bar{\nu}_{s}$. Hence

$$
P\left(\nu_{e} \rightarrow \bar{\nu}_{e}\right)=P\left(\nu_{e} \rightarrow \bar{\nu}_{\mu}\right)=P\left(\nu_{e} \rightarrow \bar{\nu}_{\tau}\right)=\frac{s^{2} c^{2}}{9} \sim 10^{-2}
$$

where the energy of $\bar{\nu}_{\alpha}$ is only $1 / 2$ that of $\nu_{e}$ and $x=0$ has been assumed. This is in principle detectable especially since the $\bar{\nu}_{e} p$ capture cross section is about 100 times that of $\nu_{e} e$ scattering at a few MeV. Unfortunately, the Super-Kamiokande experiment has an energy threshold of $6.5 \mathrm{MeV}$ for the recoil electron and taking into account the additional 1.8 $\mathrm{MeV}$ threshold for the $\bar{\nu}_{e} p \rightarrow e^{+} n$ reaction, this would require the original $\nu_{e}$ energy to be 
above 16.6 MeV, placing it outside the solar neutrino spectrum. With the recently lowered Super-Kamiokande energy threshold of $5.5 \mathrm{MeV}$, the fraction of solar $\nu_{e}$ above $14.6 \mathrm{MeV}$ is $1.6 \times 10^{-4}$. Given the small probability of $P\left(\nu_{e} \rightarrow \bar{\nu}_{e}\right)$, this will not change appreciably the total number of observed $e$-like events. Regardless of energy threshold, the inability of Super-Kamiokande to distinguish $e^{+}$from $e^{-}$or to detect the $2.2 \mathrm{MeV}$ photon from neutron capture on free protons makes it difficult to pin down this possibility in any case.

In the Sudbury (SNO) neutrino experiment 19], the energy threshold for detecting recoil electrons is $5 \mathrm{MeV}$, but since there is also a threshold of about $4 \mathrm{MeV}$ for breaking up the deuterium nucleus into two neutrons and a positron, the neutrino energy required is more than about $18 \mathrm{MeV}$. This again places it outside the solar neutrino spectrum. On the other hand, if the experimental energy threshold can be significantly lowered, then SNO may be able to see this effect because the $\bar{\nu}_{e}$ signature $\left(\bar{\nu}_{e}+d \rightarrow n+n+e^{+}\right)$is distinct from that of $\nu_{e}$

The best chance for detecting antineutrinos from the decay of $\nu_{4}$ is offered by the BOREXINO experiment 20] with a very low energy threshold of $0.25 \mathrm{MeV}$. Taking into account the 1.8 MeV needed for inverse beta decay, i.e. $\bar{\nu}_{e} p \rightarrow e^{+} n$, this means that solar neutrinos with energy above 4.1 MeV can be detected as antineutrinos. The idea of looking for antineutrinos from the sun was motivated by the possibility of a large neutrino magnetic moment which may convert $\nu_{e}$ into $\bar{\nu}_{e}$ in the sun's magnetic field. The capability of BOREXINO for detecting this has been discussed earlier 21]. For our new distinctive effect of $\nu_{4}$ decay, the observed antineutrino energy spectrum is predicted to go from $f(E)$ to $f(E / 2)$, where $E$ is the energy of the original neutrino.

For atmospheric neutrinos, since $\bar{\nu}_{\mu}$ and $\bar{\nu}_{e}$ are produced together with $\nu_{\mu}$ and $\nu_{e}$ in about equal amounts, it is not possible to tell if a given event comes from the primary neutrino or its decay product, even if the detector could measure the charge of the observed lepton. 
To search for the $\nu_{\mu} \rightarrow \bar{\nu}_{e}$ transition in the LSND and KARMEN experiments, one would use the monoenergetic $(29.8 \mathrm{MeV}) \nu_{\mu}$ from $\pi^{+}$decay at rest, which has the signature of a monoenergetic positron of $13.1 \mathrm{MeV}$ from inverse beta decay, i.e. $\bar{\nu}_{e} p \rightarrow e^{+} n$, in coincidence with a $2.2 \mathrm{MeV}$ photon from the subsequent capture of the neutron by a free proton. However, this signal is overwhelmed by the neutral-current reaction $\nu^{12} C \rightarrow \nu{ }^{12} C^{*}$, with the subsequent emission of a $15.1 \mathrm{MeV}$ photon.

In proposed long-baseline $\nu_{\mu} \rightarrow \nu_{\tau}$ appearance experiments, the oscillation probability is given by

$$
P_{\mu \tau}=\left(1-\frac{s^{2}}{3}\right)^{2}-\frac{1}{2}\left(1-\frac{2 s^{2}}{3}\right)\left(1+\cos \frac{\Delta m_{23}^{2} L}{2 E}\right)+\frac{x^{2} s^{4}}{9},
$$

which is not easily distinguished from the $s=0$ case. However, the decay products of $\nu_{4}$, i.e. $\bar{\nu}_{e}, \bar{\nu}_{\mu}$, and $\bar{\nu}_{\tau}$, may be observable with their own unique signatures, depending on the capabilities of the proposed detectors.

In the case of four-neutrino oscillations, the effective number of neutrinos $N_{\nu}$ in Big Bang Nucleosynthesis is an important constraint [22]. In this model, with $m_{4} \sim$ few $\mathrm{eV}$ and $s^{2} \sim$ few percent, the presence of a stable $\nu_{s}$ would have counted as an extra neutrino species, making $N_{\nu}=4$. This may not be acceptable if $N_{\nu}<4$, as indicated from the observed primordial ${ }^{4} \mathrm{He}$ abundance 23]. The decay of $\nu_{4}$ changes $N_{\nu}$ to $3+$ the contribution of the Majoron (i.e. 4/7).

With $\nu_{4}$ as a component of $\nu_{e}$, neutrinoless double decay has an effective $\nu_{e}$ mass of $\left(s^{2} / 3\right) m_{4} \sim 0.2 \mathrm{eV}$ if $m_{4} \sim 5 \mathrm{eV}$. This value is just at the edge of the most recent experimental upper bound 24].

Finally a comment on the neutrino contribution to dark matter may be in order. With $\nu_{4}$ decaying and $m_{1}, m_{2}$, and $m_{3}$ being too small, there is no neutrino dark matter. However, it is possible that $m_{1} \simeq m_{2} \simeq m_{3} \simeq$ few $\mathrm{eV}$, while $m_{4}$ is higher by another few $\mathrm{eV}$, in which case $\nu_{1}, \nu_{2}$, and $\nu_{3}$ will contribute to dark matter. Our discussion goes through almost 
unchanged, except that $m_{4}^{2}$ in Eq. (8) will be replaced by $m_{4}^{2}-m_{1,2,3}^{2}$.

In conclusion, we have shown in this paper that a hierarchical four-neutrino scenario is acceptable as a solution to all present neutrino data regarding the disappearance and appearance of $\nu_{e}$ and $\nu_{\mu}$. The assumed singlet neutrino of a few eV may decay into a linear combination of the three known doublet neutrinos with half of the energy. This new feature allows our proposal to be tested in future solar neutrino experiments such as BOREXINO (and perhaps SNO), and should be considered in forthcoming long-baseline accelerator neutrino experiments.

\section{ACKNOWLEDGEMENT}

One of us (G.R.) thanks the Physics Department, University of California, Riverside, for hospitality while this work was done. The research of E.M. was supported in part by the U. S. Department of Energy under Grant No. DE-FG03-94ER40837. 


\section{References}

[1] Y. Fukuda et al., Phys. Lett. B433, 9 (1998); B436, 33 (1998); Phys. Rev. Lett. 81, 1562 (1998); 82, 2644 (1999).

[2] R. Davis, Prog. Part. Nucl. Phys. 32, 13 (1994); P. Anselmann et al., Phys. Lett. B357, 237 (1995); B361, 235 (1996); J. N. Abdurashitov et al., Phys. Lett. B328, 234 (1994); Y. Fukuda et al., Phys. Rev. Lett. 77, 1683 (1996); 81, 1158 (1998); 82, 1810 (1999); 82, 2430 (1999).

[3] C. Athanassopoulos et al., Phys. Rev. Lett. 75, 2650 (1995); 77, 3082 (1996); 81, 1774 (1998).

[4] See for example E. Ma and P. Roy, Phys. Rev. D52, R4780 (1995); N. Gaur, A. Ghosal, E. Ma, and P. Roy, Phys. Rev. D58, 071301 (1998).

[5] Y. Chikashige, R. N. Mohapatra, and R. D. Peccei, Phys. Lett. 98B, 265 (1981).

[6] L. Wolfenstein, Phys. Rev. D17, 2369 (1978); S. P. Mikheyev and A. Yu. Smirnov, Sov. J. Nucl. Phys. 42, 913 (1986).

[7] N. Okada and O. Yasuda, Int. J. Mod. Phys. A12, 3669 (1997); S. M. Bilenky, C. Giunti, and W. Grimus, Eur. Phys. J. C1, 247 (1998); V. Barger, S. Pakvasa, T. J. Weiler, and K. Whisnant, Phys. Rev. D58, 093016 (1998).

[8] F. Dydak et al., Phys. Lett. 134B, 281 (1984).

[9] B. Achkar et al., Nucl. Phys. B434, 503 (1995).

[10] L. A. Ahrens et al., Phys. Rev. D31, 2732 (1985).

[11] L. Borodovsky et al., Phys. Rev. Lett. 68, 274 (1992). 
[12] A. Romosan et al., Phys. Rev. Lett. 78, 2912 (1997).

[13] G. B. Gelmini and M. Roncadelli, Phys. Lett. 99B, 411 (1981).

[14] Y. Nambu, Phys. Rev. Lett. 4, 380 (1960); J. Goldstone, Nuovo Cimento 19, 154 (1961); J. Goldstone, A. Salam, and S. Weinberg, Phys. Rev. 127, 965 (1962).

[15] V. Barger, J. G. Learned, S. Pakvasa, and T. J. Weiler, Phys. Rev. Lett. 82, 2640 (1999).

[16] P. Lipari and M. Lusignoli, Phys. Rev. D60, 013003 (1999).

[17] V. Barger, J. G. Learned, P. Lipari, M. Lusignoli, S. Pakvasa, and T. J. Weiler, hepph/9907421.

[18] T. E. Jannakos, hep-ex/9908043; for the earlier KARMEN results, see B. Zeitnitz et al., Prog. Part. Nucl. Phys. 32, 351 (1994).

[19] A. B. McDonald, in Particle Physics and Cosmology, Proc. of the 9th Lake Louise Winter Institute, 1994, edited by A. Astbury et al. (World Scientific, Singapore, 1995), p. 1.

[20] R. S. Raghavan, Science 267, 45 (1995).

[21] R. S. Raghavan et al., Phys. Rev. D44, 3786 (1991).

[22] See for example S. M. Bilenky, C. Giunti, W. Grimus, and T. Schwetz, hep-ph/9804421.

[23] C. J. Copi, D. N. Schramm, and M. S. Turner, Phys. Rev. D55, 3389 (1997); N. Hata, G. Steigman, S. Bludman, and P. Langacker, Phys. Rev. D55, 540 (1997); K. A. Olive and D. Thomas, hep-ph/9811444.

[24] L. Baudis et al., hep-ex/9902014. 


\begin{tabular}{|c|c|c|c|}
\hline$m_{4}^{2}\left(\mathrm{eV}^{2}\right)$ & $\Gamma_{4} / m_{4}$ & $f(\mathrm{LSND})$ & $f(\mathrm{E} 776)$ \\
\hline 4.8 & 0 & 3.92 & 0.04 \\
\hline 5.0 & 0.030 & 3.04 & 0.04 \\
\hline 5.2 & 0.065 & 2.21 & 0.19 \\
\hline 5.4 & 0.085 & 1.72 & 0.38 \\
\hline 5.6 & 0.095 & 1.37 & 0.57 \\
\hline 5.8 & 0.095 & 1.09 & 0.78 \\
\hline 6.0 & 0.086 & 0.54 & 1.03 \\
\hline 6.2 & 0.068 & 0.55 & 1.37 \\
\hline 6.4 & 0.038 & 0.22 & 1.94 \\
\hline 6.6 & 0 & 0.0 & 3.0 \\
\hline
\end{tabular}

Table 1: Null solution for oscillation and decay at the two CDHSW detector distances. 